\title{
Materials Express
}

\section{Synthesis and cytotoxic evaluation of gum arabic surface modified cadmium telluride quantum dots}

\author{
Uzunuigbe O. Edwina ${ }^{1,4, *}$, Ayabei Kiplagat ${ }^{2,3}$, Nicole R. S. Sibuyi ${ }^{3}$, Mervin Meyer ${ }^{3}$, \\ Abidemi Paul Kappo', and Martin O. Onani ${ }^{2,3}$ \\ ${ }^{1}$ Biotechnology and Structural Biology (BSB) Group, Department of Biochemistry and Microbiology, \\ University of Zululand, KwaDlangezwa 3886, South Africa \\ ${ }^{2}$ Department of Chemistry, University of the Western Cape, Private BagX17, Bellville, 7535, South Africa \\ ${ }^{3}$ DST/Mintek Nanotechnology Innovation Centre Department of Biotechnology, University of the Western Cape, \\ Private BagX17, Bellville, 7535, South Africa \\ ${ }^{4}$ Rubber Research Institute of Nigeria, Iyanomo, PMB 1049, Benin City, Edo State, Nigeria
}

\begin{abstract}
Water-soluble cadmium telluride (CdTe) quantum dots (QDs) were capped with gum Arabic (GA) is a non-toxic, water-soluble glycoprotein polymer commonly used in the food and pharmaceutical industries. The GA was used to stabilise cadmium telluride quantum dots (GA-QDs) and provides functional groups for other molecules such as nucleic acids, peptides and antibodies to be attached to the QDs for biological and biomedical applications. In this study, the GA was used to cap and stabilise QDs using two different methods. These QDs were characterised using Ultraviolet-visible (UV-vis) and Photoluminescence (PL) spectroscopy, X-powder ray diffraction (XRD), High-resolution transmission electron microscopy, zeta potential and particle size distributions. Cytotoxicity of these QDs was also investigated using four different human cell lines; HeLa, MCF-7, PC-3 and U87 cancer cells. The QDs-MPA was capped with 3-mercaptopropionic acid, QDs-GA2 was stabilized and capped with GA at $60{ }^{\circ} \mathrm{C}$ for two hours, and QDs-GA12 was stabilized and capped with GA for twelve hours at room temperature $\left(25^{\circ} \mathrm{C}\right)$ with continuous stirring; These QDs were found to be highly luminescent with $\mathrm{PL}$ values of $675 \mathrm{~nm}, 678 \mathrm{~nm}$ and $677 \mathrm{~nm}$ respectively. The average polydispersity index (PDI) were $0.36 \pm 0.02$, $0.27 \pm 0.02,0.35 \pm 0.01$ for QDs-MPA, QDs-GA2 and QDs-GA12, respectively. The average particles size from HRTEM, XRD and hydrodynamic size showed that the QDs-GA have bigger particles sizes; $(56.12 \mathrm{~nm} \pm 1.14)$, $(68.69 \mathrm{~nm} \pm 2.08)$ and $(77.85 \mathrm{~nm} \pm 1.69)$ for QDs-MPA, QD-GA2 and QD-GA12 respectively. Cytotoxicity studies of these QDs were carried out using WST-1 cell proliferation assay on four different tumour cell line. The results showed that these cells were over 50 per cent viable and the QDs-GA capped had higher cell percentage viability.
\end{abstract}

Keywords: Gum Arabic (GA), Quantum Dots (QD), Cadmium Telluride (CdTe), Cancer Cells, Cytotoxicity.

\section{INTRODUCTION}

Semiconductor nanoparticles have been widely explored based on novelties and their unique properties [1-4]. Semiconductor nanocrystal or QDs have been of great interest [5-6], and their synthesis has also improved

\footnotetext{
*Author to whom correspondence should be addressed.
}

in recent years. This may be attributed to the development in the instrumentation and the broad application of QDs in different fields [7-11]. Amid the numerous forms of QDs, the cadmium telluride (CdTe) QDs has found full application in industrial and biomedical applications [12]. These CdTe QDs shows exceptional use in nanobiotechnology for imaging of cells because of their 
unique characteristics which include; small size range of 1-10 nm [6, 8, 13], photoluminescence, narrow emission which is size-dependent, photo-stability and relatively high quantum yield $[5,12-16]$. They are also unique in the sense that they can be excited by a single wavelength and emit different emit light of a different wavelength which is determined by the size of the QDs [5, 13, 16-17]. The method used to synthesize CdTe and material used as a capping agent of CdTe is essential because it determines its application. QDs are made up of the inner core and a shell which help to increase their optical property and stability $[5,18]$. These exceptional features make them a uniquely outstanding choice as fluorescent probes for biological applications, cellular bio-imaging, and diagnosis, especially in cancer research $[6,13,19]$. Gum Arabic (GA) is an edible glycoprotein polymer from acacia plants that is non-toxic and odourless. It is a heterogeneous polymer of the arabinogalactan type made up of carboxyl and amine groups. It is primarily used in the food and pharmaceutical industries as an emulsifier and a stabilizer [20-23]. In the synthesis of nanoparticles, the GA can provide functional groups which may act as a reducing agent, give colloidal stability $[12,24-25]$ and as a capping agent to the nanoparticles.

Most recently, the biological research focal point is shifting to using natural materials for synthesizing nanoparticles, which are characteristically non-toxic to the metabolic system [26-30]. The use of GA for surface modification is termed as green surface modification and gives numerous advantages such as being economical, safer and produce less waste with minimal risk [18, 31-33]. Cell-based assays are commonly used for screening compounds or substances that have effects on cell proliferation or show direct cytotoxic effects that can ultimately lead to the death of cells [34]. These assays are quantitative, sensitive, and reliable colorimetric assay that measures viability, proliferation, and activation of cells. [35]. Various types of this assay are available in vitro like \{3-(4,5-dimethylthiazol-2-y1)-2,5diphenyl tetrazolium bromide (MTT), WST-1 (Watersoluble tetrazolium (WST) proliferation assay) [36].

Although the use of natural plant material in the synthesis of nanoparticle has been termed as safe, there is evidence that human get in contact with nanoparticles in various forms; dermal contact, inhalation, and ingestion hence there is need to ascertain their toxicity, and that is to establish a concentration threshold of these substances $[2,37]$. Reference [36] in their studies used CHO cell line to detect bacillus species toxins produce from food.

In this research, we are reporting for the first time the use of gum Arabic polymer to cap and stabilize CdTe quantum dots using two different routes. The GA maintains the integrity of the quantum dots and enhances its applicability.

\section{EXPERIMENTAL PROCEDURES}

\subsection{Chemicals}

The reagents were used as purchased from the manufacturers without any additional purification. Cadmium chloride $\left(\mathrm{CdCl}_{2}\right)$, 3-mercaptopropionic acid (MPA, $99 \%$ ), sodium borohydride $\left(\mathrm{NaBH}_{4}\right)$, tellurium $(99.8 \%)$, zinc acetate (99.9\%), thiourea (99\%), ethanol (99\%) and acetone were purchased from Sigma-Aldrich, and were used without further purification. Cell Proliferation Reagent 4-[3-(4-iodophenyl)-2-(4-nitrophenyl)-2H5-tetrazolio]-1,3-benzene disulfonate (WST-1) (Roche Diagnostics, Mannheim, Germany), RPMI-1640, Penicillin and Streptomycin, Sterile phosphate buffer, Trypsin, Dulbecco's modified Eagle's medium (DMEM) was from gotten from Lonza, FBS (Fetal bovine serum) obtained from Gibco life technologies and 96-well flat-bottom transparent microplate (Greiner) and the Gum Arabic (GA) obtained from the Rubber Research Institute of Nigeria (RRIN).

UV-visible and photoluminescence of the QDs were carried out using Polar Star Omega Spectrophotometer (BMG Lab tech) and Nano log HORIBA FL3-22-TRIAX, respectively. The sizes and structural patterns of QDs were studied by carrying out powder X-ray diffraction (XRD) analysis to supplement the HRTEM estimations. Powder XRD patterns were recorded at room temperature using a Bruker AXS D8 Advance diffractometer equipped with $\mathrm{Cu}-\mathrm{K} \alpha$ radiation and nickel-filtered $(\lambda=$ $1.5418 \AA$ ) at $40 \mathrm{kV}$ and $40 \mathrm{~mA}$. The powder X-ray patterns were collected by varying two thetas $(2 \theta)$ from 15-65 . High-Resolution Transmission Electron Microscope (model TECNAIF3OST-TEM) was used to determine the morphology and the elemental distribution of the QDs; the sample was prepared by putting a drop of the QDs solution on a nickel grid and allowing to dry it before analysis. Fourier transform infrared (FTIR) analysis was carried out on a Perkin-Elmer spectrum100 series instrument using potassium bromide $(\mathrm{KBr})$ pellets and prepared with the dry sample of the QDs. The hydrodynamic size distribution and the zeta potential of the QDs were measured using a Malvern Zeta sizer Nano-ZS (Malvern, Worcestershire, UK).

\subsection{Synthesis and Characterization of CdTe-QDs}

The synthesis of the CdTe was carried out using methods described previously $[17,38-40]$ but with some slight modifications [41-43]. All reactions were carried out under an inert atmosphere using nitrogen gas. All the flasks were washed, rinsed with acetone and dried in the oven before synthesis. Sodium hydrogen Telluride complex (NaHTe) prepared by dissolving 1 mmol sodium borohydride $\left(\mathrm{NaBH}_{4}\right)$ and $0.04 \mathrm{mmol}$ tellurium powder (Te) in deionized water. The solution was heated at $80^{\circ} \mathrm{C}$ for 30 minutes with continuous stirring, which resulted in a colour change from black to the purple colour solution of NaHTe. The Cd/MPA precursor prepared by mixing 
$0.4 \mathrm{mmol} \mathrm{CdCl}_{2}$ and $0.6 \mathrm{mmol} \mathrm{MPA}$ in deionized water. The initial $\mathrm{pH}$ of 2.48 was raised to $\mathrm{pH} 11.7$ and the solution heated to $80{ }^{\circ} \mathrm{C}$ under inert atmospheric conditions in a three-neck flask. The NaHTe solution was injected into the flask. The reaction maintained at $100{ }^{\circ} \mathrm{C}$ for 2 hours allowing the growth of CdTe nanocrystals. Afterwards, the solution was rapidly cooled down on the ice, and $2 \mathrm{~mL}$ $0.1 \mathrm{M}$ zinc acetate and $0.1 \mathrm{M}$ thiourea in deionized water was added to the CdTe solution and which raise the $\mathrm{pH}$ to 11.5. The solution was degassed and subsequently heated to $90{ }^{\circ} \mathrm{C}$ for 1 hour in a three-neck flask which allowed the growth of MPA-capped CdTe QDs. The solution ultimately changed colour from purple to green/yellow. The solution was aliquot in $2 \mathrm{~mL}$ Eppendorf tubes and centrifuged for 3 minutes at a speed of $13000 \mathrm{rpm}$ using Eppendorf mini spin plus microcentrifuge to remove impurities and unreacted materials. The supernatant collected in sterile $15 \mathrm{~mL}$ tubes, and the pellet discarded.

\subsection{Preparation of QDs-GA2 and QD-GA12 Using Gum Arabic Polymer}

This was carried out according to the method by [44]. The gum arabic (GA) capped CdTe QDs was prepared by adding $1 \mathrm{~mL}$ of (5\%) GA solution to $5 \mathrm{~mL}$ of MPA-capped CdTe QDs and stirred for 12-15 hours at room temperature $25{ }^{\circ} \mathrm{C}$ to produce QDs-GA12. QDs-GA2 was stirred for two hours at $60{ }^{\circ} \mathrm{C}$ after the addition of $1 \mathrm{~mL} 5 \% \mathrm{GA}$ solution was added to $5 \mathrm{~mL}$ of QD solution. The samples were recovered by addition of absolute ethanol in a $15 \mathrm{~mL}$ tube, the resulting solution centrifuged at $14000 \mathrm{rpm}$ for $5 \mathrm{mins}$, the supernatant discarded, and the pellet re-suspended in distilled water for further characterisation.

\subsection{Investigation of the Effect of CdTe QDs in Cancer Cells}

The cytotoxicity of CdTe QDs on selected cancer cell lines was assessed using WST-1 \{4-[3-(4-iodophenyl)-2(4-nitrophenyl)-2H-5-tetrazolio]-1, 3-benzene disulfonate in-vitro assay. The four cell lines: (cervical cancer) HeLa, (breast cancer) MCF-7, (prostate cancer) PC-3 and (brain cancer) U-87 were from ATCC. MCF-7, HeLa and U-87 cells were cultured in Dulbecco's modified Eagle's medium (DMEM) and PC-3 cells in RPMI-1640 (BioWhattaker, Lonza) that was supplemented with $10 \%$ FBS (Fetal bovine serum) (Gibco life technologies) and $1 \%$ Penicillin and $50 \mathrm{mg} / \mathrm{mL}$ of Streptomycin (BioWhattaker, Lonza). The cells were grown at $37{ }^{\circ} \mathrm{C}$ in a humidified atmosphere with $5 \% \mathrm{CO}_{2}$ in a Shel $\mathrm{Lab}$ incubator until $\sim 70 \%$ confluence.

After that, cells were seeded $(100 \mu \mathrm{L}$ per well $)$ at a density of $1 \times 10^{5}$ cells per $\mathrm{mL}$ in a 96 well plate and cultured for 24 hours at $37^{\circ} \mathrm{C}$. After 24 hours of incubation, the cells were treated for another 24 hours with different concentrations of QDs-MPA, QD-GA2 and QD-GA12 and the positive control cells with $6 \%$ DMSO. After the treatment, the spent media replaced with $100 \mu \mathrm{L}$ fresh media. $10 \mu \mathrm{L}$ of WST-1 reagent was added into each well and allow to incubate at $37{ }^{\circ} \mathrm{C}$ for 4 hours and absorbance measured at $440 \mathrm{~nm}$ and $630 \mathrm{~nm}$ a using BMG Labtech Polar Star Omega microplate reader. The percentage cell viability calculated as the percentage mean absorbance ratio of treated cells against percentage means, absorbance ratio of untreated cells multiplied by 100 as shown in the formula below:

$$
\text { Percentage }(\%) \text { Cell viability }=\frac{\text { treated cells }}{\text { untreated cells }} \times 100
$$

\section{RESULTS AND DISCUSSION}

\subsection{Optical Characterization}

The QDs solutions were centrifuged immediately after synthesis to remove impurities and unreacted materials. The PL and UV were used to monitor and confirm formation of the CdTe QDs, and GA Capped nanoparticles respectively. The purified nanocrystal band gap was determined by using Tauc's plot, as shown in the Figure 1(b) below. The UV-vis absorption spectra of the QDs-MPA was about $466 \mathrm{~nm}$ while for QD-GA2, and QD-GA12 was $453 \mathrm{~nm}$ and $455 \mathrm{~nm}$, respectively as seen Figure 1(a). The tangent was extrapolated to cut the $x$-axis, and the bandgap was determined to be $2.05 \mathrm{eV}$. The value falls within the theoretical range of semiconductors which is between 0 and $3.5 \mathrm{eV}$. This is indicative of blue shift effect because the particle sizes from TEM analysis is within the range of $2-30 \mathrm{~nm}$.

The PL wavelengths and peaks are in agreement with other studies $[12,15]$. Photoluminescence peak showed a red shift in emission spectra. PL spectra of the QDs-MPA and the GA-capped QDs was in the same range of 676 to $678 \mathrm{~nm}$. When PL peaks were overlaid, the QD-GA12 had the highest peak intensity, as shown in Figure 2. This is probably due to the bigger particle size of QD-GA12 from TEM analysis $(3.9 \mathrm{~nm})$ and hydrodynamic size $(77.85 \mathrm{~nm})$.

The full width at half maxima (FWHM) of particles increases with incubation time, and this depends on the increase in the size of particles [12]. This was observed in QDs-GA12 quantum dots with the largest particle sizes and FWHM values for PL spectra, then QDs-GA2 and QDs-MPA with the following values; $103.5 \mathrm{~nm}, 93.5 \mathrm{~nm}$ and $70 \mathrm{~nm}$ respectively; this is consistent with the increase in size of the QDs, with QDs-GA12 having the most significant particle size.

\subsection{X-ray Diffraction (XRD) Powder Analysis}

This XRD was used to ascertain the crystal nature of the QDs. XRD powder analysis pattern of QDs-MPA, QDsGA2 and QDs-GA12 are shown in Figure 3 below.

Figure 3 shows a major distinctive broad peak at $2 \theta$ of 26.7, 26.5 and 26.52 corresponding to the indices (498), (304) and (782) respectively. The two minor small peaks 

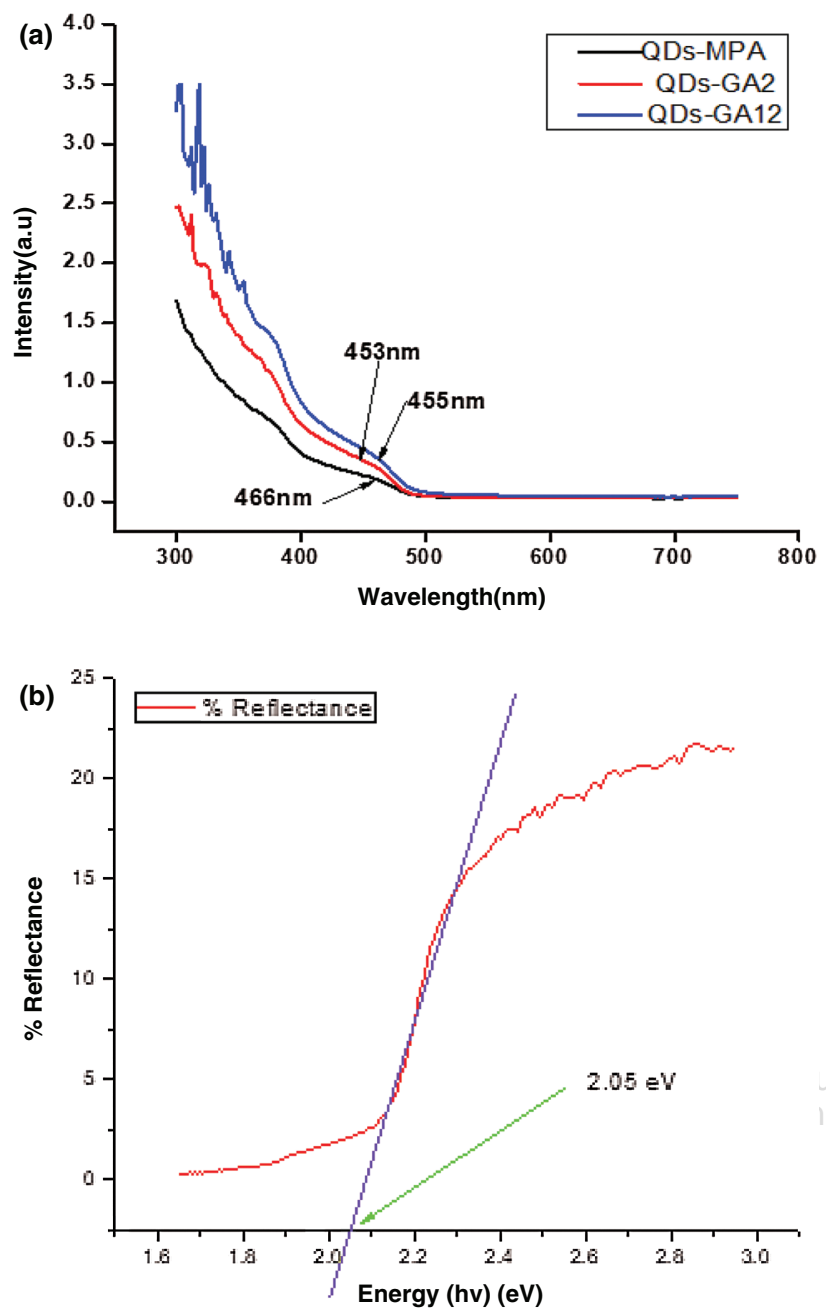

Fig. 1. (a) UV-vis spectra of the QDs-MPA, QDs-GA2 and QDsGA12. (b) The representative tauc plot of the purified CdTe nanocrystals.

correspond to $2 \theta$ degree; 43.88, 52.02 for QDs-MPA; 43.67, 52.03 for QDs-GA2 and 43.98, 51.91 for QDsGA12 as shown in (Fig. 3). The crystalline cubic phase of the QDs was established, and this corresponds to the cubic CdTe standard pattern (JCPDS card no: 01-0752083), similar to the work of [45]. Broad distinctive peaks imply the presence of small-sized nanoparticles $[9,12]$.

The Derby Scherrer equation was also used to calculate the crystallite size of the quantum dots from the XRD pattern

$$
\text { Particle Size }(S)=\frac{B \lambda}{\beta \cos \theta}
$$

Where $S$ is the size of particles (nm), $\lambda=1.5406 \AA$ is a constant which is the wavelength of the $\mathrm{X}$-ray radiation, $B=0.91, \beta$ is full width at half maximum (FWHM) of the $\mathrm{XRD}$ pattern, $\theta$ is the Bragg's angle in degree. The average sizes of the different quantum dots using XRD peaks were; 3.78, 3.97 and $4.2 \mathrm{~nm}$ for QDs-MPA, QDs-GA2, and QDs-GA12 respectively as shown in Figure 3, these

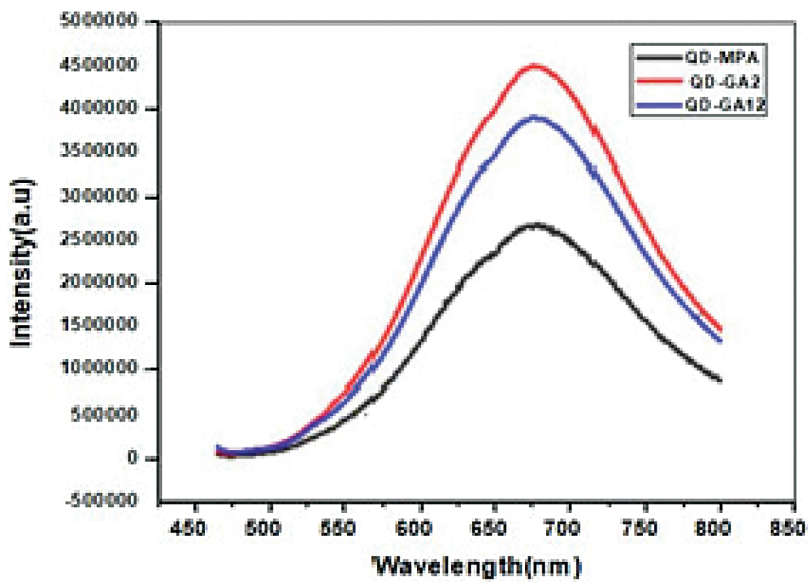

Fig. 2. The photoluminescence spectrum of the QDs-MPA, QDsGA2, and QDs-GA12.

values measure up with the previous data obtained for particle sizes using image $\mathrm{J}$. This observation was established with HRTEM and particle size analysis.

\subsection{High-Resolution Transmission \\ Electron Microscope}

Morphologically, the CdTe QDs were characterised using HRTEM to determine shape, size and structure. The HRTEM results are shown in Figures 4(a), (b)-6(a), (b).

Image $\mathrm{J}$ software was used on the HRTEM images to ascertain diameter of the QDs; Average size of the particle was $2.7 \mathrm{~nm}, 3.45 \mathrm{~nm}$ and $3.9 \mathrm{~nm}$ for QDs-MPA, QDs-GA2, and QDs-GA12 respectively. The particle size distribution from image $J$ is presented in Figures 4(a)-6(a) for each of the QDs. These QDs were well dispersed from HRTEM images. EDS was used for elemental analysis of the individual elements present in the nanoparticles. The spectra showed traces of cadmium, tellurium, carbon, oxygen, zinc, sodium and chlorine. Research has

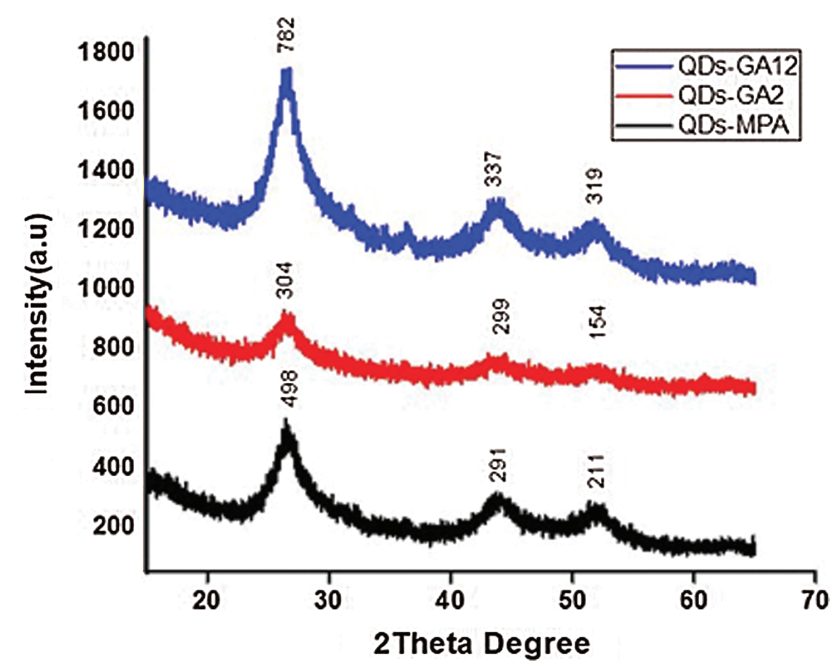

Fig. 3. XRD pattern of the QDs-MPA, QD-GA2, and QD-GA12. 


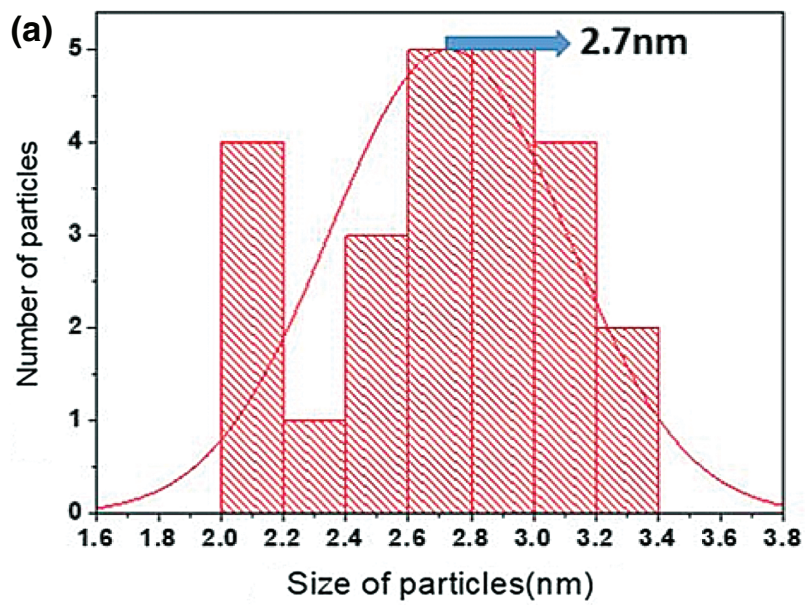

(b)

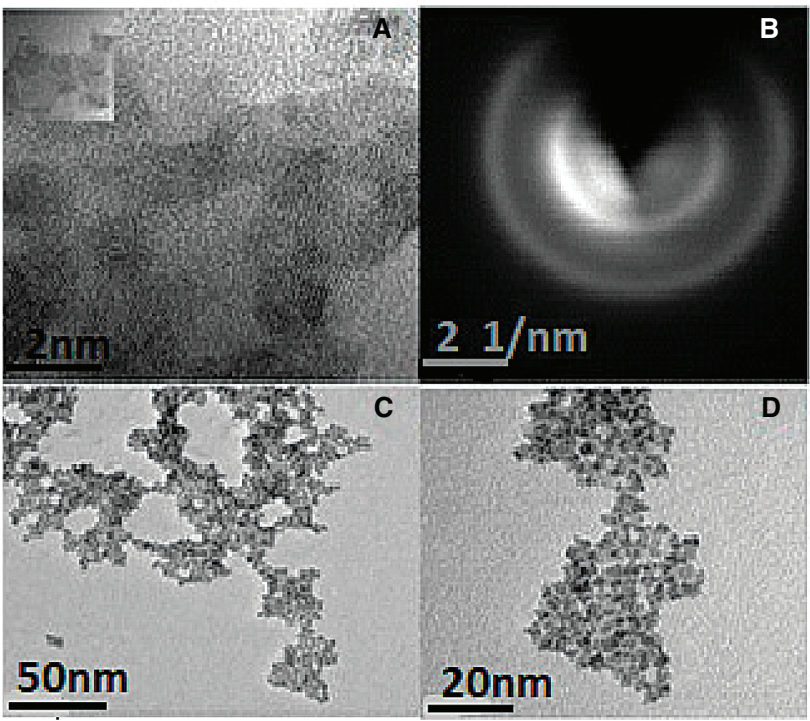

Fig. 4. (a) The particle size distribution of QDs-MPA. (b) HRTEM and SAED images of QDs-MPA.

shown that particles capped with polymer tend to have bigger sizes compared to MPA capped particles as seen QDs-MPA and QDs-GA nanoparticles. This result is consistent with the literature [24]. Also, there was a significant difference in the size of the QDs capped for 2 hours and 12 hours; QDs-GA2 and QDs-GA12 respectively, this may probably be due to the effect of temperature on the quantum particles. The HRTEM and SAED images of QDs-MPA, QDs-GA2, and QDs-GA12, are shown in Figures 4(b)-6(b) respectively.

\subsection{Fourier Transform Infrared Spectroscopy (FTIR)} FTIR analysis displayed the functional groups that are present in the compound. This was measured from the range of 4000 to $350 \mathrm{~cm}^{-1}$ and was used to confirm the capping of QD with GA polymer, as shown in Figure 7.

The FTIR spectra of the GA powder, QDs-MPA, QDsGA2, and QDs-GA12 were analyzed, overlaid. FTIR

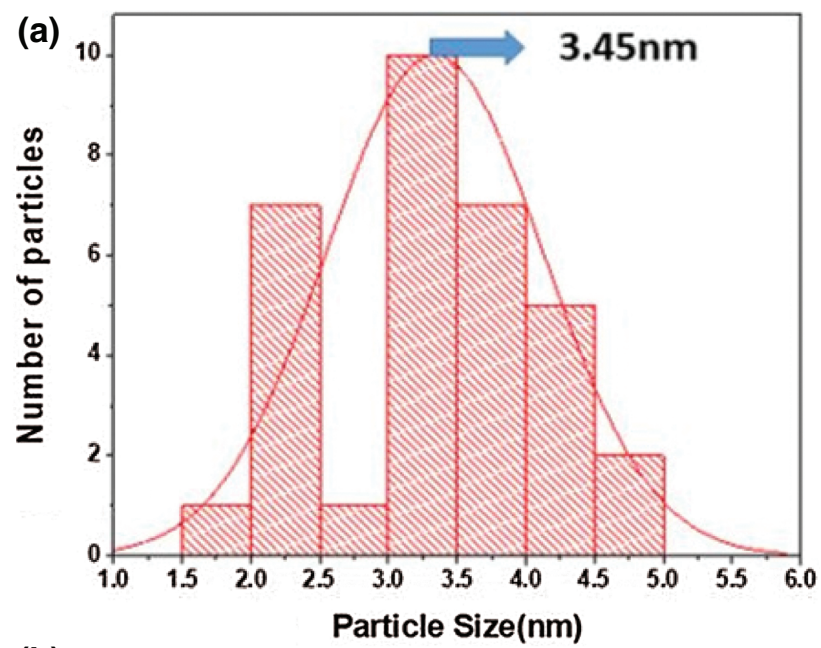

(b)

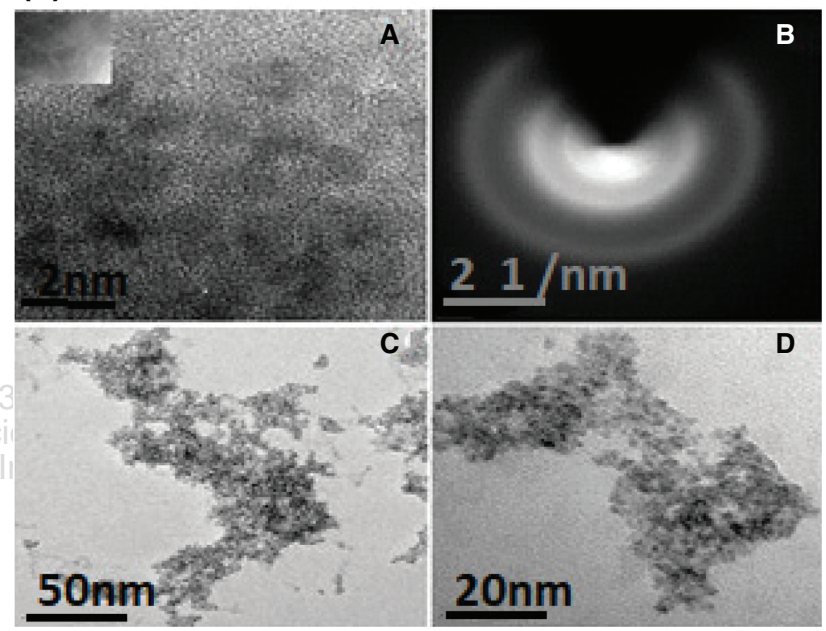

Fig. 5. (a) The particle size distribution of QDs-GA2. (b) HRTEM and SAED images of QDs-GA2.

spectra of GA powder and QD-GA2 and QD-GA12 as depicted in Figure 7 showed similar peaks confirming the capping of the CdTe QDs, the characteristic stretch at $3300 \mathrm{~cm}^{-1}$ to $3328 \mathrm{~cm}^{-1}$ for $-\mathrm{OH}$ stretching is attributed to the monosaccharide which makes up the GA polymer possessing some free-OH, [46-47] the band at $2917 \mathrm{~cm}^{-1}$ stretching is $\mathrm{R}-\mathrm{CH}_{2}-\mathrm{R}$ is found in the GA capped QDs and GA powder. The stretch between $995 \mathrm{~cm}^{-1}$ and $1047 \mathrm{~cm}^{-1}$ are commonly referred to as GA "fingerprint" and represents the $\mathrm{C}=\mathrm{O}$ and $\mathrm{C}-\mathrm{N}$ frequencies stretching. There was also aliphatic amine stretching vibration of the arabinogalactan chains of GA [48] at $1290 \mathrm{~cm}^{-1}$ which is a characteristic band of aliphatic amine. The broad QDs stretch (Fig. 7) between $3340 \mathrm{~cm}^{-1}$ to $3200 \mathrm{~cm}^{-1}$ is $\mathrm{O}-\mathrm{H}$ associated with acid and the sharp peak at $1700 \mathrm{~cm}^{-1}$ is assigned to a carboxylic acid (R-COOH) associated with the capping Mercaptopropionic acid (MPA) [49]. Surface chemistry of nanoparticles has some crucial impact on their application biologically. The zeta potential of nanoparticles is a vital marker for predicting the long-term stability of 


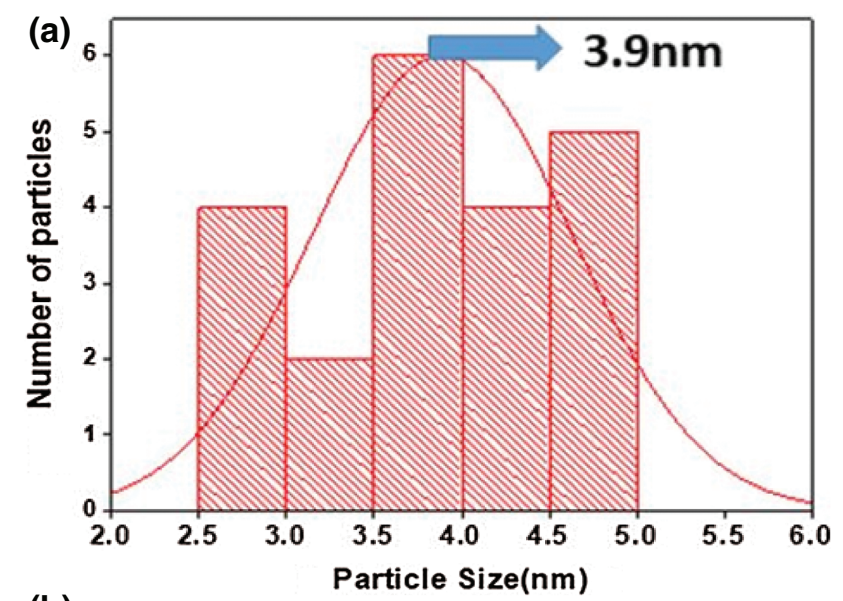

(b)

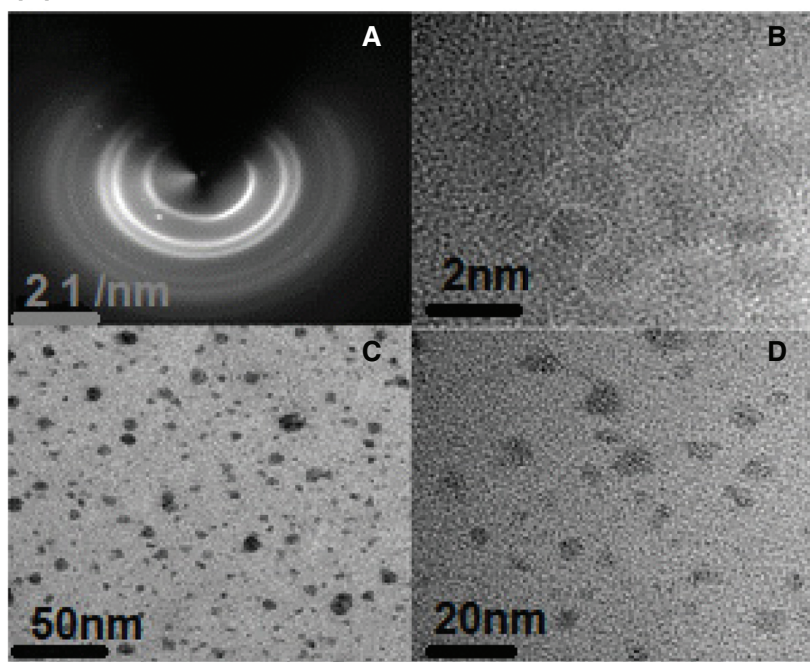

Fig. 6. (a) The particle size distribution of QDs-GA12. (b) HRTEM and SAED images of QDs-GA12.

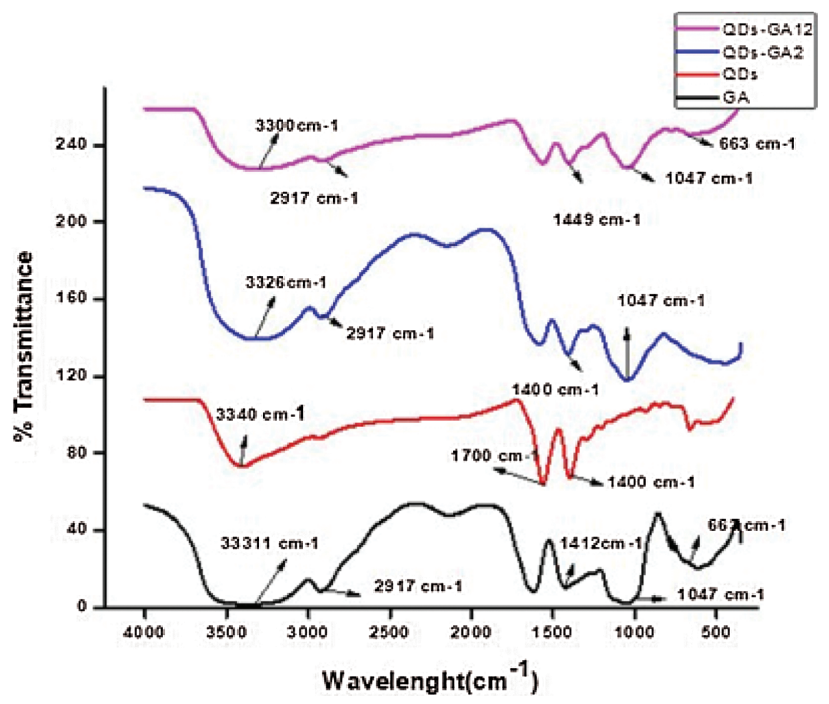

Fig. 7. The FT-IR plot of QDs-MPA, QDs-GA2, and QDs-GA12. nanoparticles in solution and also to determine the surface charge and a high zeta potential values of about $\pm 25 \mathrm{mV}$ to $\pm 60 \mathrm{mV}$ which indicates that the particle is stable [50]. The hydrodynamic particle size and polydispersity index (PDI) of the QDs was also measured. Measurements were done in triplicate at room temperature at $25^{\circ} \mathrm{C}$, and the average value obtained. The bar chat distribution of the Zeta potential, PDI and hydrodynamic sizes of the quantum dots are shown in Figure 8 with the bars having different alphabet indicating that they are significantly different from each other.

Zeta potential is a vital measurement or parameter used to ascertain long-term stability and analytical assessment of nanoparticles [51]. Zeta potential of the quantum dots was not significantly different from each other and with a mean value of $(34.12 \pm 0.8),(30.87 \pm 1.18)$ and
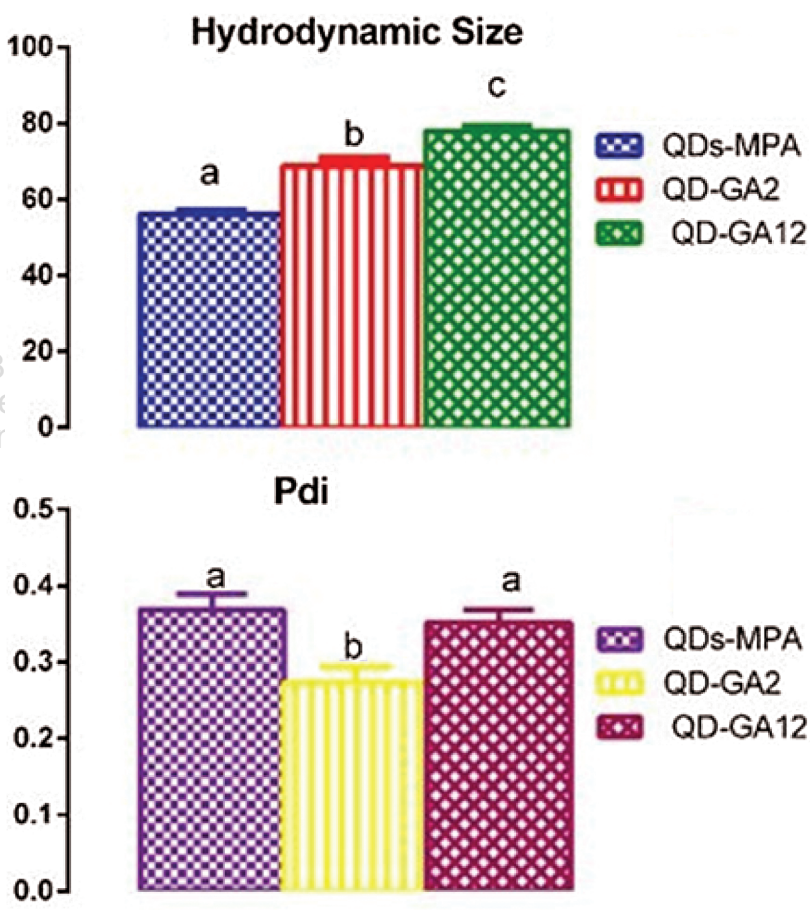

\section{Zeta potential}

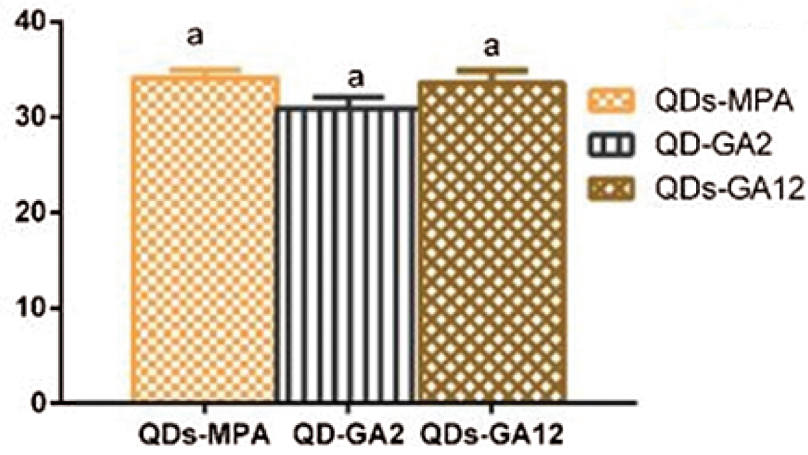

Fig. 8. Histogram showing the zeta potential, polydispersity index (PDI) and hydrodynamic distribution of the QDs-MPA, QDs-GA2, and QDs-GA12. 


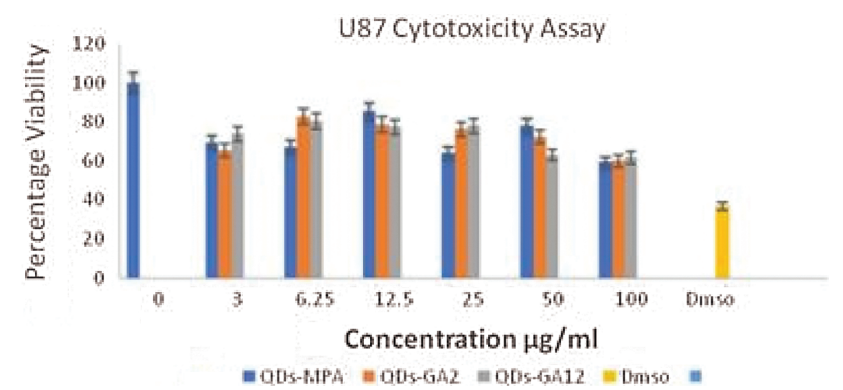

Fig. 9. Bar chart showing U87 cytotoxicity assay using QDs-MPA, QDs-GA2 and QDs-GA12.

(33.56 \pm 1.3$)$, Particles with zeta potential value of above \pm 30 are said to be very stable according to the literature [52]. The zeta potential of the QDs was above \pm 30 with QD-GA12 having the highest zeta value and QD-GA2 having the lowest zeta potential. The number of particle ratio to the total number of particles present in a solution is termed polydispersity index. The colloid solution with low PDI values of less than one for the particles is said to be monodispersed [53]. The mean PDI values of QDs were $(0.37 \pm 0.02),(0.27 \pm 0.02)$ and $(0.35 \pm 0.01)$ for QDs-MPA, QDs-GA2 and QDs-GA12, respectively. The PDI values of QDs-MPA and QDs-GA12 were not significantly different from each other but differed over $p$ values $(P<0.05)$. The hydrodynamic size of the three quantum dots showed significant differences in their sizes with $p$ values of $(P>0.0001)$ with a mean value of $(56.12 \mathrm{~nm} \pm$ $1.14),(68.69 \mathrm{~nm} \pm 2.08)$ and $(77.85 \mathrm{~nm} \pm 1.69)$ for QDsMPA, QDs-GA2, and QDs-GA12 respectively.

QDs capped with GA polymer had a larger hydrodynamic size which was probably due to the effect of the capping agent. Zeta potential is an essential parameter for ascertaining the long and short-term stability of a material. This substance with high zeta potential values are said to be highly stable and low values tends to coagulate or agglomerate, making them unstable. The zeta potential of the QDs were $(34.12 \pm 0.8),(30.87 \pm 1.18)$ and $(33.56 \pm 1.3)$ for QDs-MPA, QDs-GA2 and QDs-GA12,

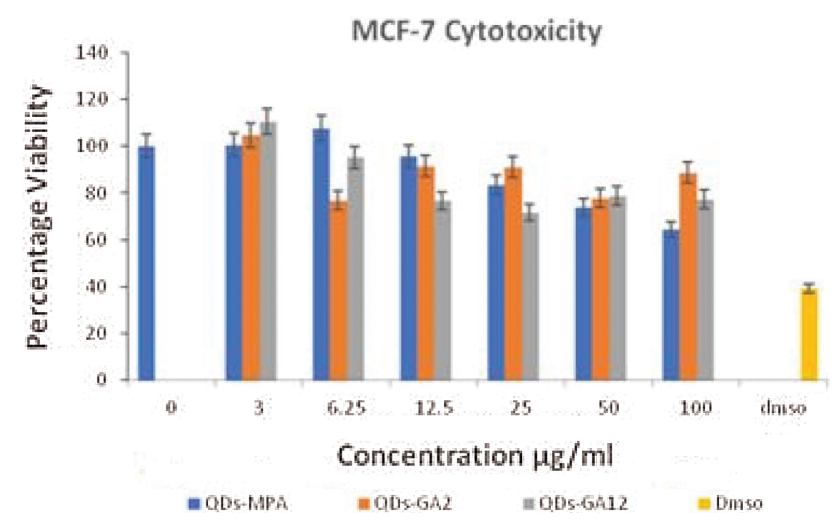

Fig. 10. Bar chart showing MCF-7 cytotoxicity assay using QDsMPA, QDs-GA2 and QDs-GA12.

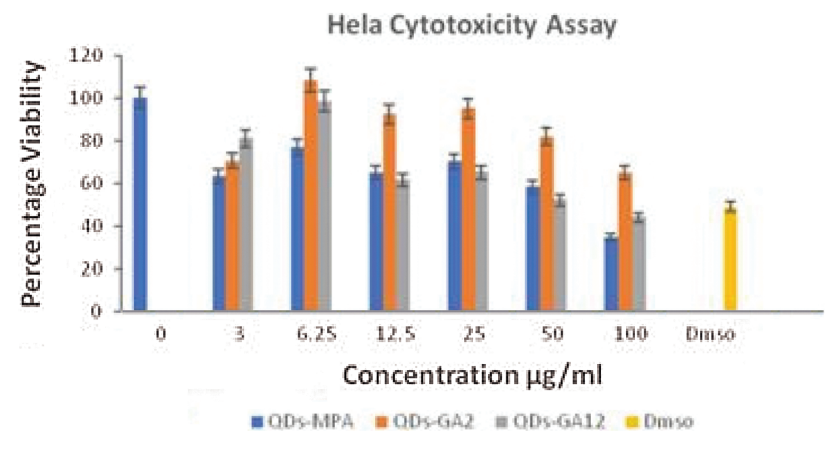

Fig. 11. The bar chart is showing HeLa cytotoxicity assay using QDsMPA, QDs-GA2 and QDs-GA12.

respectively. QDs-MPA and QDs-GA12 have high-value zeta potential, which implies these particles were very stable.

\subsection{Cytotoxicity Studies of the Bare and GA Capped Quantum Dots}

Nanoparticles capped with polymers are presumed to be less toxic when compared to those capped with synthetic materials because of the displayed stabilities. Research has shown that water-soluble quantum dots have a low level of toxicity. Figures 9-12 showed the cytotoxicity of the MPA and GA capped QDs on selected cancer cell lines after 24-hour exposure.

The results showed that the QDs have over 50 percent viability for all the cell lines. Literature has shown that quantum dots coated with polymer shows excellent colloidal stability in solution [54-56] and PC-3 cells were very viable for all the concentrations except at $100 \mu \mathrm{g} / \mathrm{ml}$ for MPA capped QDs and QDs-GA2, this is following the previous studies of [57] in literature. Surface coating plays a vital role in the cytotoxicity of quantum dots $[38,58]$. All other cell lines were over 50\% viable when compared with control 6\% DMSO used in the experiment as a control treatment, and the toxicity is dose-dependent. Also, for all the cell line, especially HeLa and MCF-7, we notice there was an increase in cell viability at $6.25 \mu \mathrm{g} / \mathrm{ml}$ concentrations probably due to an increase in cell proliferation.

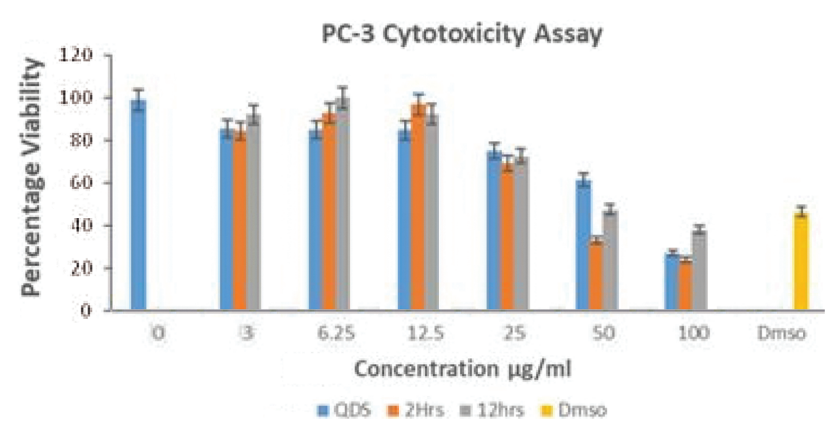

Fig. 12. The bar chart showing PC-3 cytotoxicity assay using QDsMPA, QDs-GA2 and QDs-GA12. 


\section{CONCLUSION}

In this experiment Luminescent CdTe QDs capped with GA was synthesised and capped at two different temperatures [60 degrees for 2 hours (QDs-GA2) and at room temperature $\left(25^{\circ} \mathrm{C}\right)$ for 12 hours with continuous stirring (QDs-GA12). Capping of nanoparticles with other materials can help to stabilise the particle and provide functional groups. QDs-GA12 were found to be very stable and monodispersed. Their cytotoxicity was investigated using four different cell lines, and the cells were found to be over 50 percent viable; this implies that these QDs have low toxicity, and it was in a concentration-dependent manner. Highly luminescent quantum dots have found applicability biologically in in-vivo cell bioimaging and cellular targeting; hence, QDs synthesised from these studies with their characteristic features, and low toxicity can be applicable for cell bioimaging purpose.

Acknowledgment: The research reported in this article was supported through funding by NRF and the support of the University of Zululand Research committee. The University of the Western Cape (UWC) is greatly acknowledged.

\section{References and Notes}

1. Zhang, H., Zhou, Z., Yang, B. and Gao, M., 2003. The influence of carboxyl groups on the photoluminescence of mercaptocarboxylic acid-stabilized CdTe nanoparticles. The Journal of Physical Chemistry B, 107(1), pp.8-13.

2. Grabowska-Jadach, I., Zuchowska, A., Olesik, M., Drozd, M., Pietrzak, M., Malinowska, E. and Brzozka, Z., 2018. Cytotoxicity studies of selected cadmium-based quantum dots on $2 \mathrm{D}$ versus $3 \mathrm{D}$ cell cultures. New Journal of Chemistry, 42(15), pp.12787-12795.

3. Madkour, L.H., 2019. Environmental impact of nanotechnology and novel applications of nano materials and nano devices. Nanoelectronic Materials, 116, pp.605-699.

4. Wang, R., Lu, K.Q., Tang, Z.R. and Xu, Y.J., 2017. Recent progress in carbon quantum dots: Synthesis, properties and applications in photocatalysis. Journal of Materials Chemistry A, 5(8), pp.37173734

5. Nguyen, K.C., Willmore, W.G. and Tayabali, A.F., 2013. Cadmium telluride quantum dots cause oxidative stress leading to extrinsic and intrinsic apoptosis in hepatocellular carcinoma HepG2 cells. Toxicology, 306, pp.114-123.

6. Wu, X., Liu, H., Liu, J., Haley, K.N., Treadway, J.A., Larson, J.P., Ge, N., Peale, F. and Bruchez, M.P., 2003. Immunofluorescent labeling of cancer marker Her2 and other cellular targets with semiconductor quantum dots. Nature Biotechnology, 21(1), pp.41-46.

7. Jamieson, T., Bakhshi, R., Petrova, D., Pocock, R., Imani, M. and Seifalian, A.M., 2007. Biological applications of quantum dots. Biomaterials, 28(31), pp.4717-4732.

8. Smith, A.M., Duan, H., Mohs, A.M. and Nie, S., 2008. Bioconjugated quantum dots for in vivo molecular and cellular imaging. Advanced Drug Delivery Reviews, 60(11), pp.1226-1240.

9. Kyobe, J.W., Mubofu, E.B., Makame, Y.M., Mlowe, S. and Revaprasadu, N., 2016. Cadmium sulfide quantum dots stabilized by castor oil and ricinoleic acid. Physica E: Low-dimensional Systems and Nanostructures, 76, pp.95-102.

10. Ramirez, H.Y., Flórez, J. and Camacho, Á.S., 2015. Efficient control of coulomb enhanced second harmonic generation from excitonic transitions in quantum dot ensembles. Physical Chemistry Chemical Physics, 17(37), pp.23938-23946.
11. Owen, J. and Brus, L., 2017. Chemical synthesis and luminescence applications of colloidal semiconductor quantum dots. Journal of the American Chemical Society, 139(32), pp.10939-10943.

12. Bao, H., Hao, N., Yang, Y. and Zhao, D., 2010. Biosynthesis of biocompatible cadmium telluride quantum dots using yeast cells. Nano Research, 3(7), pp.481-489.

13. Weng, J., Song, X., Li, L., Qian, H., Chen, K., Xu, X., Cao, C. and Ren, J., 2006. Highly luminescent CdTe quantum dots prepared in aqueous phase as an alternative fluorescent probe for cell imaging. Talanta, 70(2), pp.397-402.

14. Walling, M., Novak, J. and Shepard, J.R., 2009. Quantum dots for live cell and in vivo imaging. International Journal of Molecular Sciences, 10(2), pp.441-491.

15. Li, L., Qian, H., Fang, N. and Ren, J., 2006. Significant enhancement of the quantum yield of CdTe nanocrystals synthesized in aqueous phase by controlling the $\mathrm{pH}$ and concentrations of precursor solutions. Journal of Luminescence, 116(1-2), pp.59-66.

16. McHugh, K.J., Jing, L., Behrens, A.M., Jayawardena, S., Tang, W., Gao, M., Langer, R. and Jaklenec, A., 2018. Biocompatible semiconductor quantum dots as cancer imaging agents. Advanced Materials, 30(18), p.1706356.

17. Mushonga, P., Onani, M.O., Madiehe, A.M. and Meyer, M., 2013. One-pot synthesis and characterization of InP/ZnSe semiconductor nanocrystals. Materials Letters, 95, pp.37-39.

18. Şenel, B., Demir, N., Büyükköroğlu, G. and Yıldız, M., 2019 Graphene quantum dots: Synthesis, characterization, cell viability, genotoxicity for biomedical applications. Saudi Pharmaceutical Journal, 27, pp.846-858.

19. Rakovich, A. and Rakovich, T., 2018. Semiconductor versus graphene quantum dots as fluorescent probes for cancer diagnosis and therapy applications. Journal of Materials Chemistry B, 6(18), pp. 2690-2712.

20. Kattumuri, V., Katti, K., Bhaskaran, S., Boote, E.J., Casteel, S.W., Fent, G.M., Robertson, D.J., Chandrasekhar, M., Kannan, R. and Katti, K.V., 2007. Gum arabic as a phytochemical construct for the stabilization of gold nanoparticles: In vivo pharmacokinetics and X-ray-contrast-imaging studies. Small, 3(2), pp.333-341.

21. Montenegro, M.A., Boiero, M.L., Valle, L. and Borsarelli, C.D. 2012. Gum Arabic: More than an edible emulsifier. in Products and Applications of Biopolymers. London, UK, Intech Open Publisher.

22. Kaddam, L., Fadl-Elmula, I., Eisawi, O.A., Abdelrazig, H.A., Salih, M.A., Lang, F. and Saeed, A.M., 2017. Gum arabic as novel antioxidant agent in sickle cell anemia, phase II trial. BMC. Hematology, 17(1), p.4.

23. Duran, H., Alkan, F.Ü., Ulkay, M.B., Karakuş, S., Aktaş, A. and Şişmanoğlu, T., 2019. Investigation of the in vitro cytotoxic effects and wound healing activity of ternary composite substance (hollow silica sphere/gum arabic/methylene blue). International Journal of Biological Macromolecules, 121, pp.1194-1202.

24. Zhang, L., Yu, F., Cole, A.J., Chertok, B., David, A.E., Wang, J. and Yang, V.C., 2009. Gum arabic-coated magnetic nanoparticles for potential application in simultaneous magnetic targeting and tumor imaging. The American Association of Pharmaceutical Scientists Journal, 11(4), pp.693-699.

25. Djajadisastra, J.S., Purnamasari, P. and Pujiyanto, A.N.U.N.G., 2014 Antioxidant activity of gold nanoparticles using gum arabic as a stabilizing agent. International Journal of Pharmacy and Pharmaceutical Sciences, 6(7), pp.462-465.

26. Kang, B., Opatz, T., Landfester, K. and Wurm, F.R., 2015. Carbohydrate nanocarriers in biomedical applications: Functionalization and construction. Chemical Society Reviews, 44(22), pp.8301-8325.

27. Shombe, G.B., Mubofu, E.B., Mlowe, S. and Revaprasadu, N., 2016. Synthesis and characterization of castor oil and ricinoleic acid capped CdS nanoparticles using single source precursors. Materials Science in Semiconductor Processing, 43, pp.230-237. 
28. Shombe, G.B., Mubofu, E.B., Mlowe, S. and Revaprasadu, N., 2016. Synthesis of hierarchical $\mathrm{PbS}$ nanostructures capped with castor oil. Materials Letters, 185, pp.17-20.

29. Kyobe, J.W., Mubofu, E.B., Makame, Y.M., Mlowe, S. and Revaprasadu, N., 2016. The use of castor oil and ricinoleic acid in lead chalcogenide nanocrystal synthesis. International Nano Letters, 6(4), pp.235-242.

30. Kyobe, J.W., Mubofu, E.B., Makame, Y.M., Mlowe, S. and Revaprasadu, N., 2015. CdSe quantum dots capped with naturally occurring biomasses oils. New Journal of Chemistry, 39(9), pp.7251-7259.

31. Bhosale, R.R., Kulkarni, A.S., Gilda, S.S., Aloorkar, N.H., Osmani, R.A. and Harkare, B.R., 2014. Innovative ecofriendly approaches for green synthesis of silver nanoparticles. International Journal of Pharmaceutical Sciences and Nanotechnology, 7, pp.2328-2337.

32. Rauwel, P., Küünal, S., Ferdov, S. and Rauwel, E., 2015. A review on the green synthesis of silver nanoparticles and their morphologies studied via TEM. Advances in Materials Science and Engineering, 2015, 9 pages.

33. Yakout, S.M. and Mostafa, A.A., 2015. A novel green synthesis of silver nanoparticles using soluble starch and its antibacterial activity. International Journal of Clinical and Experimental Medicine, 8(3), p.3538.

34. Riss, T.L., Moravec, R.A., Niles, A.L., Duellman, S., Benink, H.A., Worzella, T.J. and Minor, L., 2016. Cell viability assays. in Assay Guidance Manual [Internet]. Bethesda MD, USA, Eli Lilly and Company and the National Center for Advancing Translational Sciences.

35. Senthilraja, P. and Kathiresan, K., 2015. In vitro cytotoxicity MTT assay in vero, HepG2 and MCF-7 cell lines study of marine yeast. Journal of Pharmaceutical Sciences, 5(3), pp.080-084.

36. Ngamwongsatit, P., Banada, P.P., Panbangred, W. and Bhunia, A.K., 2008. WST-1-based cell cytotoxicity assay as a substitute for MTT-based assay for rapid detection of toxigenic bacillus species using CHO cell line. Journal of Microbiological Methods, 73(3), pp.211-215.

37. Judson, R., Houck, K., Martin, M., Richard, A.M., Knudsen, T.B., Shah, I., Little, S., Wambaugh, J., Woodrow Setzer, R., Kothya, P. and Phuong, J., 2016. Editor's Highlight: Analysis of the effects of cell stress and cytotoxicity on in vitro assay activity across a diverse chemical and assay space. Toxicological Sciences, 152(2), pp.323-339.

38. Kiplagat, A., Sibuyi, N.R., Onani, M.O., Meyer, M. and Madiehe, A.M., 2016. The cytotoxicity studies of water-soluble $\mathrm{InP} / \mathrm{ZnSe}$ quantum dots. Journal of Nanoparticle Research, 18(6), pp.147-148.

39. Oluwafemi, S.O., Revaprasadu, N. and Ramirez, A.J., 2008. A novel one-pot route for the synthesis of water-soluble cadmium selenide nanoparticles. Journal of Crystal Growth, 310(13), pp.3230-3234.

40. Dunpall, R., Mlowe, S. and Revaprasadu, N., 2014. Evidence of oriented attachment in the growth of functionalized ZnTe nanoparticles for potential applications in bio-imaging. New Journal of Chemistry, 38(12), pp.6002-6007.

41. Chomoucka, J., Drbohlavova, J., Majzlikova, P., Prasek, J., Pekarek, J., Hrdy, R. and Hubalek, J., 2013. Conjugation Bovine Serum Albumin with CDTE Quantum Dots. Conference Proceedings 5th International Conference Nanocon, Vol. 1, pp.566-571.

42. Li, Z.J., Li, X.B., Wang, J.J., Yu, S., Li, C.B., Tung, C.H. and Wu, L.Z., 2013. A robust "artificial catalyst" in situ formed from CdTe QDs and inorganic cobalt salts for photocatalytic hydrogen evolution. Energy and Environmental Science, 6(2), pp.465-469.

43. Yang, P., Zhang, A., Sun, H., Liu, F., Jiang, Q. and Cheng, X., 2010. Highly luminescent quantum dots functionalized and their conjugation with $\mathrm{IgG}$. Journal of Colloid and Interface Science, 345(2), pp.222-227.

44. Kim, J., Park, Y., Yoon, T.H., Yoon, C.S. and Choi, K., 2010 Phototoxicity of $\mathrm{CdSe} / \mathrm{ZnSe}$ quantum dots with surface coatings of 3-mercaptopropionic acid or tri- $n$-octylphosphine oxide/gum arabic in daphnia magna under environmentally relevant UV-B light. Aquatic Toxicology, 97(2), pp.116-124.

45. Arivarasan, A., Sasikala, G. and Jayavel, R., 2014. In situ synthesis of CdTe: CdS quantum dot nanocomposites for photovoltaic applications. Materials Science in Semiconductor Processing, 25, pp.238-243.

46. Vasile, F.E., Martinez, M.J., Ruiz-Henestrosa, V.M.P., Judis, M.A. and Mazzobre, M.F., 2016. Physicochemical, interfacial and emulsifying properties of a non-conventional exudate gum (Prosopis alba) in comparison with gum arabic. Food Hydrocolloids, 56, pp.245-253.

47. Quintanilha, R.C., Orth, E.S., Grein-Iankovski, A., Riegel-Vidotti, I.C. and Vidotti, M., 2014. The use of gum Arabic as "Green" stabilizer of poly(aniline) nanocomposites: A comprehensive study of spectroscopic, morphological and electrochemical properties. Journal of Colloid and Interface Science, 434, pp.18-27.

48. Paulsen, Z., Onani, M.O., Allard, G.R., Kiplagat, A., Okil, J.O., Dejene, F.B. and Mahanga, G.M., 2016. The effect of varying the capping agent of magnetic/luminescent $\mathrm{Fe}_{3} \mathrm{O}_{4}-\mathrm{InP} / \mathrm{ZnSe}$ core-shell nanocomposite. Physica B: Condensed Matter, 480, pp.156-162.

49. Haider, M.J. and Mehdi, M.S., 2014. Study of morphology and Zeta potential analyzer for silver nanoparticles. International Journal of Scientific and Engineering Research, 5(7), pp.381-385.

50. Tantra, R., Schulze, P. and Quincey, P., 2010. Effect of nanoparticle concentration on zeta-potential measurement results and reproducibility, Particuology, 8(3), pp.279-285.

51. Sharma, S., Shukla, P., Misra, A. and Mishra, P.R., 2014. Interfacial and colloidal properties of emulsified systems: Pharmaceutical and biological perspective. Colloid and Interface Science in Pharmaceutical Research and Development, pp.149-172.

52. Elbagory, A., Cupido, C., Meyer, M. and Hussein, A., 2016 Large scale screening of Southern African plant extracts for the green synthesis of gold nanoparticles using microtitre-plate method. Molecules, 21(11), pp.1498-1517.

53. Fernández-Argüelles, M.T., Yakovlev, A., Sperling, R.A. Luccardini, C., Gaillard, S., Sanz Medel, A., Mallet, J.M., Brochon, J.C., Feltz, A., Oheim, M. and Parak, W.J., 2007. Synthesis and characterization of polymer-coated quantum dots with integrated acceptor dyes as FRET-based nanoprobes. Nano Letters, 7(9), pp.2613-2617.

54. Tomczak, N., Liu, R. and Vancso, J.G., 2013. Polymer coated quantum dots. Nanoscale, 5(24), pp.12018-12032.

55. Xiong, H.M., 2010. Photo luminescent $\mathrm{ZnO}$ nanoparticles modified by polymers. Journal of Materials Chemistry, 20(21), pp.4251-4262.

56. Soenen, S.J., Demeester, J., De Smedt, S.C. and Braeckmans, K., 2012. The cytotoxic effects of polymer-coated quantum dots and restrictions for live cell applications. Biomaterials, 33(19), pp.4882-4888

57. Dhar, R., Singh, S. and Kumar, A., 2015. Effect of capping agents on optical and antibacterial properties of cadmium selenide quantum dots. Bulletin of Materials Science, 38(5), pp.1247-1252.

58. Rajabi, H.R. and Farsi, M., 2016. Study of capping agent effect on the structural, optical and photocatalytic properties of zinc sulfide quantum dots. Materials Science in Semiconductor Processing, 48, pp.14-22.

Received: 12 June 2019. Accepted: 14 November 2019. 